\title{
OSTEOSSÍNTESES COM HASTES INTRAMEDULARES EM CRIANÇAS
}

\author{
OSTEOSYNTHESIS WITH INTRAMEDULLARY NAILS ON CHILDREN
}

Helio Jorge Alvachian Fernandes ${ }^{1}$, Eduardo Abdalla Saad ${ }^{2}$, Fernando Baldy dos Reis ${ }^{3}$

\section{RESUMO}

Os autores fazem uma ampla revisão da literatura destacando o emprego de hastes intramedulares flexíveis no tratamento de fraturas em crianças. Destacam o tratamento das fraturas diafisárias do fêmur e dos ossos do antebraço e enfatizam também a importância da abordagem não cirúrgica. A idade e o peso limite das crianças ainda não estão bem definidos para o emprego do método. A retirada de implantes tem destaque controverso na literatura, com tendência da permanência dos implantes.

Descritores - Criança; Fixação de fratura/métodos; Fixação intramedular de fratura; Fixação interna de fraturas; Fixadores internos; Dispositivos de fixação ortopédica; Titânio; Aço inoxidável

\section{INTRODUÇÃO}

As hastes intramedulares elásticas vêm sendo utilizadas, nos últimos anos, como método de tratamento de algumas fraturas em crianças. Atualmente, as exigências do mundo moderno nos levam a considerar as dificuldades para o tratamento não cirúrgico com a permanência das crianças em aparelhos gessados. Os pais trabalham, há dificuldade para cuidados domésticos, consideram-se o tempo de afastamento escolar e até mesmo questões de maior conforto dos pacientes. Assim sendo, fraturas, que há alguns anos eram tratadas incruentamente, são tratadas cirurgicamente. As fraturas cujo tratamento é feito com hastes intramedulares flexíveis são as principais indicações para hastes intramedulares, principalmente as diafisárias do fêmur e dos ossos do antebraço; no úmero e tíbia, ainda há controvérsia na literatura. O objetivo desta revisão é apresentar os aspectos atuais da utilização das hastes intramedulares para as fraturas diafisárias das crianças.

\section{ABSTRACT}

The authors present a comprehensive review of the literature emphasizing the use of flexible intramedullary nails in the treatment of fractures in children, focusing the treatment of femoral shaft and forearm fractures and emphasizing the importance of the non-surgical approach. Children's age and weight threshold are not well defined for the use of the method. The removal of implants is a controversial matter in the literature, with a trend towards keeping the implants.

Keywords - Child; Fracture fixation/methods; Fracture fixation, Intramedullary Fracture fixation, Internal; Internal fixators; Orthopedic fixation devices; Titanium; Stainless steel.

\section{Consolidação e hastes intramedulares elásticas}

As hastes intramedulares elásticas estabilizam as fraturas a distância, não abordam diretamente o foco fraturário e permitem a mobilização precoce, diminuindo o tempo de hospitalização e possibilitando o retorno mais rápido às atividades escolares. $\mathrm{O}$ princípio de tratamento é a estabilidade relativa. A consolidação é secundária, pois a estabilização permite pequenos movimentos na região da fratura aliada às características anatômicas das crianças, que apresentam o periósteo grosso e elevado poder osteogênico. A estabilidade obtida permite mobilidade articular indolor sem comprometer a redução.

\section{Princípios do tratamento}

As hastes intramedulares flexíveis funcionam como tutores que, quando pré-tensionadas e com três pontos de apoio, conferem às fraturas maior estabilidade ${ }^{(1,2)}$.

\footnotetext{
1 - Professor Afiliado e Chefe do Setor do Trauma e Pronto-Socorro da Disciplina de Traumatologia do Departamento de Ortopedia e Traumatologia da EPM/Unifesp.

2 - Doutor; Médico Assistente da Disciplina de Ortopedia Pediátrica do Departamento de Ortopedia e Traumatologia da EPM/Unifesp.

3 - Professor Livre-Docente e Chefe da Disciplina de Traumatologia do Departamento de Ortopedia e Traumatologia da EPM/Unifesp. 


\section{HASTES DE AÇO OU TITÂNIO?}

As hastes intramedulares flexíveis são disponíveis, tanto em ligas de aço quanto de titânio. A escolha envolve a preferência do cirurgião e disponibilidade do material, bem como as características físicas da liga. Estudos clínicos comparativos mostram igualdade entre ambos, com resultados semelhantes ${ }^{(3)}$. Em nosso meio, Soni et al $^{(4)}$ apresentaram um modelo computadorizado bidimensional, simulando uma fratura do fêmur em criança tratada com hastes flexíveis de aço e titânio, e avaliaram comparativamente a distribuição das tensões. Concluíram que o comportamento biomecânico foi qualitativamente melhor no modelo com hastes de titânio. $\mathrm{Na}$ análise quantitativa, restrita às regiões do foco de fratura, os valores são estatisticamente semelhantes. No estudo das deformações, observaram comportamento biomecânico mais homogêneo no modelo com hastes de titânio.

Diversos autores relataram semelhanças entre as hastes flexíveis de aço e titânio, tanto em experiências in vitro como em resultados clínicos ${ }^{(5-13)}$. O aço possui menor módulo de elasticidade, enquanto o titânio tem características elásticas mais próximas das do osso, com melhor qualidade de pré-tensionamento. Assim sendo, onde maior resistência é necessária, como fraturas em crianças no final do crescimento, fraturas onde o canal medular é mais estreito, lesão extensa de partes moles ou crianças obesas, as hastes elásticas de aço têm melhor indicação. Em pacientes que apresentam fraturas onde é necessário maior pré-tensionamento e elasticidade do implante, como as das da extremidade distal do fêmur, podem-se indicar as hastes intramedulares de titânio.

Em nosso meio, Saad ${ }^{(14)}$ apresentou resultados clínicos comparáveis aos encontrados na literatura utilizando hastes flexíveis de aço no tratamento de fraturas diafisárias de fêmur em crianças.

\section{Indicações para as hastes elásticas}

Atualmente, são utilizadas as hastes elásticas nas fraturas em crianças acima de cinco anos, principalmente nas diafisárias do fêmur ${ }^{(15-22)}$ e nas diafisárias dos ossos do antebraço ${ }^{(23-26)}$.

\section{Planejamento pré-operatório}

É muito importante a análise radiográfica das fraturas em incidências anteroposterior e perfil. Em geral, quando se utilizam duas hastes intramedulares flexíveis, calcula-se que cada haste deva ter calibre de no máximo 40\% do diâmetro da porção mais estreita do canal medular fraturado ${ }^{(27)}$. Na prática, calcula-se o diâmetro das hastes como cerca de um terço do menor diâmetro do canal medular, em geral, a região do istmo nas duas incidências. Esses detalhes são importantes, em especial nas fraturas diafisárias femorais, pois se necessita curvar as hastes dentro do canal medular. Quando são utilizadas duas hastes, estas devem ter o mesmo calibre ${ }^{(27)}$, sendo a soma do diâmetro delas cerca de 1 a $2 \mathrm{~mm}$ menor que o diâmetro do istmo. O comprimento das hastes é medido pela distância entre as placas de crescimento nas extremidades proximal e distal. Quanto à curvatura das hastes, o máximo da curva, cerca de $40^{\circ}$, deve coincidir com o nível da fratura em sua porção convexa.

Temos preferência pela mesa cirúrgica comum radiotransparente, isso porque possibilita executar manobras de redução de maneira mais livre, verificar a mobilidade do quadril e controlar mais facilmente eventuais desvios rotacionais.

\section{Técnica cirúrgica para fraturas femorais (Figura 1)}

O paciente é posicionado em mesa cirúrgica comum radiotransparente. A fratura é reduzida, se possível, por manipulação suave, sendo feito o controle radioscópico. O diâmetro das hastes é selecionado com base no planejamento pré-operatório, sendo confirmado no membro afetado antes de sua inserção.

O requisito essencial é o exato pré-tensionamento das hastes, de modo que o ápice da curva da haste esteja no local da fratura. A segunda haste de mesmo diâmetro é preparada para proporcionar uma curva diametralmente oposta no local da fratura. O diâmetro da haste deve ser semelhante para hastes de aço e titânio e de cerca de um terço do diâmetro interno do istmo do canal medular. Há autores que utilizam hastes de aço com diâmetro 1mm menor que as de titânio. Em nossa experiência isso não foi observado ${ }^{(14)}$.

A distância entre as placas de crescimento proximal e distal é medida, considerando 1 a $2 \mathrm{~cm}$ de margem de segurança entre o implante, a fise e o pré-tensionamento. Procura-se deixar a curvatura máxima ao nível da fratura para criar uma tensão que seria o segundo ponto de apoio da haste. Em regra, a inserção local será na metáfise óssea. Controle radioscópico é realizado durante a inserção das hastes. A haste selecionada é introduzida suavemente na metáfise e com a ponta angulada afastada do córtex. 
As fraturas diafisárias transversas ou oblíquas curtas em ossos longos são as com melhor indicação para o método; porém, as indicações têm-se ampliado consideravelmente com o tempo e a experiência pessoal do cirurgião.

O tratamento das fraturas diafisárias femorais em crianças varia de acordo com a idade ${ }^{(1,28)}$. Todavia, em crianças acima de cinco anos de idade, diversos autores recomendaram o tratamento cirúrgico com hastes intramedulares flexíveis, considerando sua superioridade frente ao tratamento com aparelhos gessados, com base em diversos fatores, como tempo de consolidação, retorno às atividades escolares e complicações ${ }^{(15-22)}$.

O limite de idade superior é mais difícil de determinar. Trabalhos recentes têm mostrado crescente angulação no local da fratura após a utilização de hastes intramedulares elásticas, especialmente em crianças mais velhas e mais pesadas ${ }^{(11)}$. No entanto, não existe nenhuma alternativa ideal. O risco de necrose avascular da cabeça do fêmur a partir da inserção de haste intramedular rígida em adolescentes é bem descrita ${ }^{(28)}$. Na maioria dos casos, é possível permitir mobilização precoce, com função de suporte de peso parcial, com ou sem imobilização complementar, dependendo do peso, tamanho da criança e da redução obtida ${ }^{(29)}$.

Os resultados na faixa etária de cinco a 14 anos são clinicamente satisfatórios ${ }^{(5-13)}$. O tempo de permanência hospitalar é menor, ocorrendo a consolidação em torno de oito a dez semanas ${ }^{(14)}$. Não consolidação ou retarde de consolidação são incomuns e, quando isso ocorre, parece haver relação com o uso inapropriado das técnicas cirúrgicas. As complicações são limitadas à pele ou desconforto nos locais de inserção das hastes ${ }^{(1,5)}$. Alterações no comprimento ósseo por perturbações no crescimento parecem ser mínimas, com média de sobrecrescimento do fêmur em torno de $1,2 \mathrm{~mm}^{(12)}$. Classicamente, se re-
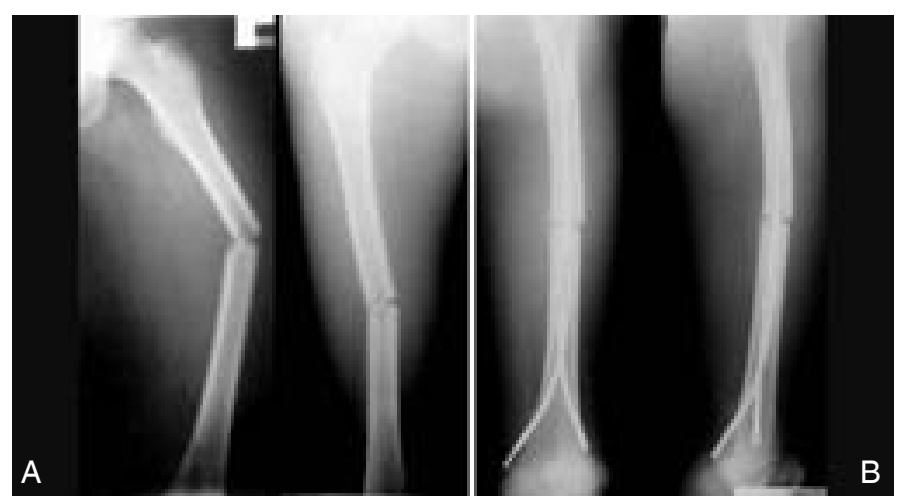

Figura 1 - A: Imagem radiográfica de fratura diafisária do fêmur em criança de 10 anos de idade. B: Imagem radiográfica da fratura, após emprego de hastes flexíveis comenda a retirada dos implantes em de quatro a seis meses, dependendo da evolução clínica e radiográfica. No entanto, alguns autores questionam a necessidade de remoção, na ausência de sintomas ${ }^{(6,7)}$.

\section{Fraturas da extremidade distal do fêmur}

A estabilização das fraturas com hastes intramedulares flexíveis pode ser realizada por via anterógrada, sendo o ponto de entrada na região lateral do fêmur 2cm abaixo do pequeno trocanter. Acredita-se que, em fraturas muito distais, hastes anterógradas estariam mais bem indicadas ${ }^{(27)}$.

\section{Pós-operatório}

A mobilização e a mobilização precoce com carga parcial são preconizadas pela maioria dos autores ${ }^{(30-40)}$, porém, alguns recomendam carga total de imediato, dependente da estabilidade obtida ${ }^{(14)}$.

\section{Fraturas de tíbia (Figura 2)}

O alargamento da extremidade proximal bem como a secção transversal triangular torna difícil a colocação de hastes simetricamente e obtenção de estabilização adequada. O tratamento não cirúrgico de fraturas da tíbia com aparelho gessado permite melhor comodidade, mobilidade e locomoção, fazendo com que a indicação para o tratamento não cirúrgico seja o de escolha. As indicações ficam restritas a situações especiais, como extensa lesão de partes moles, politraumatizados, falhas no tratamento não operatório e preferência do cirurgião.
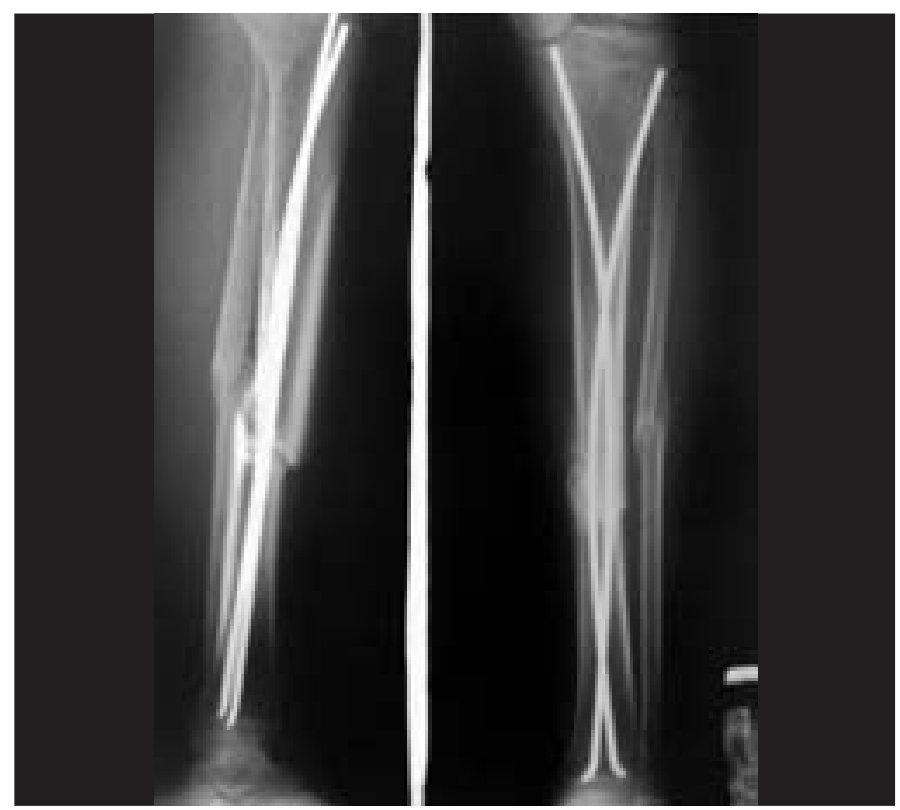

Figura 2 - Imagem radiográfica de fratura diafisária da tíbia já consolidada tratada com hastes intramedulares flexíveis 


\section{MEMBROS SUPERIORES}

\section{Úmero}

Em crianças, a maioria das fraturas da diáfise umeral pode ser tratada conservadoramente e não necessita de tratamento cirúrgico.

As fraturas da extremidade proximal do úmero que envolvem a fise, em regra, são lesões Salter-Harris tipo II e o potencial de remodelação é significativo, podendo-se aceitar alguns desvios que serão corrigidos com o crescimento ósseo. Nas fraturas instáveis ou naquelas com desvio acentuado, mais estabilidade pode ser necessária. Isso pode ser obtido por meio de fios de Kirschner inseridos através do deltoide ou pela utilização de hastes flexíveis. Duas hastes são inseridas na coluna lateral do úmero, de distal para proximal. A fise é atravessada e as pontas das hastes são impactadas na cabeça umeral. Os orifícios são feitos na coluna lateral, um acima do outro, 1-2cm de distância. Uma vez que a fratura é estabilizada, mobilização precoce pode ser iniciada, pois não existe transfixação muscular no ombro. Nesses casos, a retirada da haste é recomendada e é importante para não dificultar o crescimento da fise.

As fraturas diafisárias do úmero podem exigir estabilização com hastes flexíveis quando há falha no tratamento conservador ou na criança politraumatizada com múltiplas fraturas de ossos longos ${ }^{(8)}$. A estabilização é feita com duas hastes, sendo o ponto de entrada dependente do nível da fratura. Fraturas dos terços proximal e médio podem ser estabilizadas com duas hastes retrógradas. As fraturas do terço distal do úmero podem ser estabilizadas com duas hastes anterógradas. Ambas as hastes são inseridas através de uma entrada lateral localizada no nível da inserção do músculo deltoide. Os orifícios são feitos na cortical lateral, um acima do outro.

Quanto às fraturas supracondilianas do úmero, embora se possam utilizar hastes intramedulares flexíveis, já é consagrada a utilização de fios de Kirschner, o que torna o emprego de hastes flexíveis uma alternativa que tem vantagens questionáveis ${ }^{(9)}$.

\section{Fraturas diafisárias do antebraço}

O tratamento de fraturas do antebraço tem sido nos últimos anos realizado com hastes intramedulares flexíveis. Uma ampla variedade de dispositivos intramedulares tem sido utilizada com hastes elásticas de ti- tânio e aço ${ }^{(19-24,31)}$. A indicação é para fraturas instáveis irredutíveis quando o tratamento incruento falha ou em circunstâncias específicas, como lesões de Monteggia, fraturas da cabeça e colo do rádio.

As hastes intramedulares têm a vantagem de mínima dissecção de partes moles, sendo mais fácil e mais segura sua remoção ${ }^{(12)}$. Uma única haste é utilizada para cada osso do antebraço. Geralmente, hastes de 2,0 a 2,5mm de diâmetro são utilizadas, com o maior diâmetro empregado, se possível. A inserção é feita respeitando-se a fise proximal do rádio e cuidados devem ser tomados para evitar danos ao nervo radial superficial.

Lascombes et al $^{(13)}$ advogam que as duas hastes sejam pré-tensionadas, porém, temos observado que mesmo hastes retas são igualmente eficazes. Outra questão a saber é se ambos os ossos devem ser estabilizados. Claramente, se é apenas um osso fraturado, uma haste é necessária, porém, se ambos os ossos estão fraturados e ambas as fraturas desviadas, certamente duas hastes serão necessárias. Os casos onde as fraturas são dos dois ossos, porém apenas um apresenta desvio, devem-se reduzir e estabilizar os dois ${ }^{(14)}$ (Figura 3).

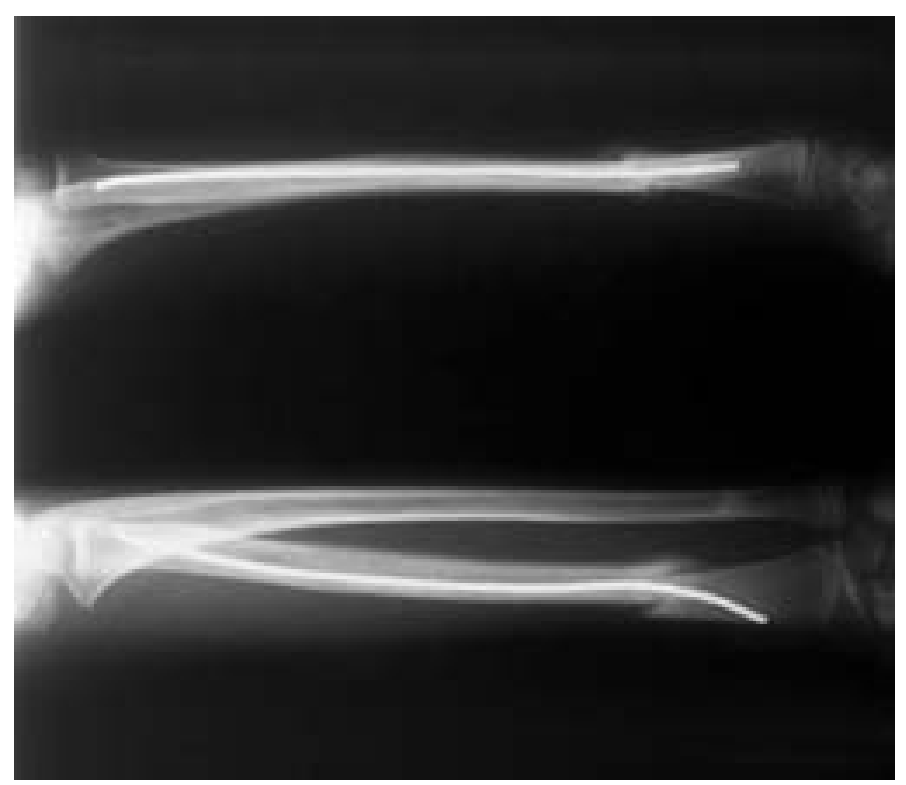

Figura 3 - Imagem radiográfica mostrando redução de fratura de Galeazzi com haste intramedular flexível que foi introduzida na metáfise distal do rádio

As fraturas do colo do rádio podem ser tratadas com hastes flexíveis inseridas na extremidade distal. A curva ponta da haste pode ser usada como um auxílio 
na redução da fratura, que é feita com manipulação suave dos fragmentos e a haste é utilizada com intuito de redução sob escopia ${ }^{(15,16)}$.

A fratura-luxação de Monteggia pode ser tratada com manipulação fechada e aplicação de um aparelho gessado ou placa e parafuso, porém, na vigência de instabilidade, a redução da cabeça radial pode ser difícil. Quando isso ocorre, uma haste flexível pode ser utilizada na ulna com o intuito de restabelecer o comprimento e facilitar a redução da cabeça do rádio.

\section{RETIRADA DOS IMPLANTES}

A retirada de implantes em crianças é um procedimento ortopédico ainda controverso. A tendência é evitar um novo procedimento cirúrgico, minimizando os riscos inerentes do ato cirúrgico bem como os custos e suas complicações. Simanovsky e Tair ${ }^{(41)}$ reavaliaram 143 crianças submetidas à remoção de implantes intramedulares do fêmur e do antebraço. Dessas, em 16 o material foi removido devido a protrusão, irritação na pele e desconforto, enquanto a maioria estava assintomática. Observaram complicações como o insucesso de retirada do material de implante em três crianças e refraturas em duas.

Não existe concordância na literatura quanto à retirada de material de implante. Diversos autores preconizam a retirada ${ }^{(42-45)}$, enquanto outros questionam essa decisão; afirmam ser necessária somente em pacientes sintomáticos ou quando o implante pode comprometer a fise pela sua flexibilidade ou porque houve necessidade de sua transfixação para a estabilidade da fratura $^{(46-49)}$.

Prefere-se retirar os implantes em crianças. Isso porque, à medida que a criança cresce, pode haver dificuldade para retirá-los, pois o implante pode ficar sepultado na cavidade medular da diáfise e há a possibilidade de refraturas, dificultando ainda mais o procedimento. A infecção por contaminação tardia do implante pode ocorrer e deve ser considerada. Por outro lado, deixar os implantes implica diminuição de custos, de novo procedimento cirúrgico e o fato de a maioria dos pacientes se encontrar assintomática.

\section{DISCUSSÃO}

A criança tem grande capacidade de remodelação óssea e, em alguns casos, o tratamento conservador resulta em deformidade ou encurtamento no local da fratura. Em muitos aspectos, as hastes intramedulares flexíveis têm indicação. O método é seguro, minimamente invasivo, tem poucas complicações e não interfere no crescimento. Além disso, está associado a curto período de hospitalização e rápido retorno à atividade de vida diária. Flynn e Waters ${ }^{(15)}$ relataram bons resultados com hastes intramedulares flexíveis no tratamento de fraturas de ossos longos em crianças, porém, ressaltaram que a maioria das fraturas pode, e deve, continuar a ser tratada com redução fechada e imobilização. Outro fator do método cirúrgico a ser considerado é a realização de nova intervenção para retirada do implante. Alguns autores questionam a necessidade de retirar o material de implante, na busca de melhores resultados, pois as maiores complicações são exatamente com a ponta das hastes salientes para facilitar a retirada; e quando se opta por não retirá-las, podem ficar com suas extremidades menos salientes, diminuindo as queixas e, consequentemente, a necessidade de um novo procedimento cirúrgico. Obviamente, tal decisão precisa ser muito bem avaliada, pois sabemos dos riscos de uma síntese perdida intramedular, caso ocorra uma nova fratura ou mesmo no caso de uma infecção óssea.

Diante dessas considerações, enfatizamos a necessidade do tratamento não cirúrgico da maioria das fraturas em crianças.

Algumas questões permanecem: qual é a idade ou peso considerado limite superior para utilização de hastes intramedulares flexíveis? É necessária a remoção dos implantes e, em caso afirmativo, quando?

É certo que as hastes intramedulares flexíveis oferecem vantagens no tratamento de algumas fraturas em crianças. Entretanto, as hastes intramedulares flexíveis destacam-se por ser o tratamento para determinadas fraturas dos ossos longos em crianças, em especial, diafisárias do fêmur e dos ossos do antebraço.

O conhecimento do método deve fazer parte do arsenal do traumatologista ortopédico, bem como o manuseio de suas complicações. 


\section{REFERÊNCIAS}

1. Luhmann SJ, Schootman M, Schoenecker PL, Dobbs MB, Gordon JE. Complications of titanium elastic nails for pediatric femoral shaft fractures. J Pediatr Orthop; 2003;23(4):443-7.

2. Orler R, Hersche O, Helfet DL, Mayo KA, Ward T, Ganz R. Avascular femur head necrosis as severe complication after femoral intramedullary nailing in children and adolescents. Unfallchirurg. 1998;101(6):495-9.

3. Vransky P, Bourdelat D, Al Faour A. Flexible stable intramedullary pinning technique in the treatment of pediatric fractures. J Pediatr Orthop. 2000;20(1):23-7.

4. Soni JF, Santili C, Lancellotti CLP, Hecke MB, Almeida FR, Karam LZ. Análise comparativa em modelo computadorizado bidimensional com simulação do emprego de hastes flexíveis de aço e titânio, na fratura do fêmur da criança, utilizando o método dos elementos finitos. Rev Bras Ortop. 2008;43(5):183-92.

5. Bar-On E, Sagiv S, Porat S. External fixation or flexible intramedullary nailing for femoral shaft fractures in children: a prospective, randomised study. J Bone Joint Surg Br. 1997;79(6):975-8,.

6. Greisberg J, Bliss MJ, Eberson CP, Solga P, d'Amato C. Social and economic benefits of flexible intramedullary nails in the treatment of pediatric femoral shaft fractures. Orthopedics. 2002;25(10):1067-70.

7. Flynn JM, Hresko T, Reynolds RA, Blasier RD, Davidson R, Kasser J. Titanium elastic nails for pediatric femur fractures: a multicenter study of early results with analysis of complications. J Pediatr Orthop. 2001;21(1):4-8.

8. Cramer KE. The pediatric polytrauma patient. Clin Orthop Relat Res.1995:;(318):125-35

9. Prevot J, Lascombes P, Metaizeau JP, Blanquart D. [Supracondylar fractures of the humerus in children: treatment by downward nailing]. Rev Chir Orthop Reparatrice Appar Mot. 1990;76(3):191-7.

10. Calder P, Achan P, Barry M. Diaphyseal forearm fractures in children treated with intramedullary fixation: outcome of $\mathrm{K}$-wire versus elastic stable intramedullary nail. Injury. 2003;34(4):278-82.

11. Nielsen $A B$, Simonsen $O$. Displaced forearm fractures in children treated with AO plates. Injury. 1984;15(6):393-6

12. Van der Reis WL, Otsuka NY, Moroz P, Mah J. Intramedullary nailing versus platefixation for unstable forearm fractures in children. J Pediatr Orthop. 1998;18(1):9-13.

13. Lascombes P, Prevot J, Ligier JN, Metaizeau JP, Poncelet T. Elastic stable intramedullary nailing in forearm shaft fractures in children: 85 cases. J Pediatr Orthop; 1990;10(2):167-71.

14. Saad EA. Avaliação clínica e radiográfica de 31 pacientes com idade entre 8 e 16 anos no tratamento de 32 fraturas do fêmur pela fixação intramedula retrógrada com hastes flexíveis de Ender [tese]. São Paulo: Universidade Federal de São Paulo, Escola Paulista de Medicina; 2003.

15. Flynn JM, Waters PM. Single-bone fixation of both-bone forearm fractures. J Pediatr Orthop;1996;16(5):655-9.

16. Gonzalez-Herranz P, Alvarez-Romera A, Burgos J, Rapariz JM, Hevia E. Displaced radial neck fractures in children treated by closed intramedullary pinning (Metaizeau technique). J Pediatr Orthop.1997;17(3):325-31

17. Metaizeau JP, Lascombes P, Lemelle JL, Finlayson D, Prevot J. Reduction and fixation of displaced radial neck fractures by closed intramedullary pinning. $J$ Pediatr Orthop. 1993;13(3):355-60.

18. Waseem M, Paton RW. Percutaneous intramedullary elastic wiring of displaced diaphyseal forearm fractures in children: a modified technique. Injury. 1999;20(1):21-4.

19. Richter D, Ostermann PAW, Ekkernkamp A, Muhr G, Hahn MP. Elastic intramedullary nailing: a minimally invasive concept in the treatment of unstable forearm fractures in children. J Pediatr Orthop. 1998;18(4):457-61.

20. Luhmann SJ, Gordon JE, Schoenecker PL. Intramedullary fixation of unstable both-bone forearm fractures in children. J Pediatr Orthop. 1998;18(4):451-6.

21. Shoemaker SD, Comstock CP, Mubarak SJ, Wenger DR, Chambers HG. Intramedullary Kirschner wire fixation of open or unstable forearm fractures in children. J Pediatr Orthop. 1999;19(3):329-37.

22. Yung SH, Lam CY, Choi KY, Ng KW, Maffulli N, Cheng JC. Percutaneous intramedullary Kirschner wiring for displaced diaphyseal forearm fractures in children. J Bone Joint Surg Br. 1998;80(1):91-4

23. Pugh DMW, Galpin RD, Carey TP. Intramedullary Steinmann pin fixation of forearm fractures in children: long-term results. Clin Orthop Relat Res. 2000;(376):39-48.

24. Street DM. Intramedullary forearm nailing. Clin Orthop Relat Res. 1986;(212):219-30.

25. Vainionpää $S$, Böstman $O$, Pätiälä $H$, Rokkanen $P$. Internal fixation of forearm fractures in children. Acta Orthop Scand. 1987;58(2):121-3.

26. Langkamer VG, Ackroyd CE. Removal of forearm plates: a review of the complications. J Bone Joint Surg Br. 1990;72(4):601-4.

27. Fricka KB, Mahar AT, Lee SS, Newton PO. Biomechanical analysis of antegrade and retrograde flexible intramedullary nail fixation of pediatric femoral fractures using a synthetic bone model. J Pediatr Orthop. 2004;24(2):167-71.

28. Ahn JL, Park JS. Pathologic fractures secondary to unicameral bone cysts. Int Orthop. 1994;18(1):20-2.

29. Roposch A, Saraph V, Linhart WE. Flexible intramedullary nailing for the treatment of unicameral bone cysts in long bones. J Bone Joint Surg Am. 2000;82(10):1447-53.

30. Knorr P, Schmittenbecher PP, Dietz HG. Elastic stable intramedullary nailing for the treatment of complicated juvenile bone cysts of the humerus. Eur J Pediatr Surg. 2003;13(1):44-9.

31. Cohen J. Intramedullary nailing for the treatment of unicameral bone cysts. J Bone Joint Surg Am. 2001;83(8):1279-80.

32. Flynn JM, Skaggs DL, Sponseller PD, Ganley TJ, Kay RM, Leitch KK. The surgical management of pediatric fractures of the lower extremity. Instr Course Lect. 2003;52:647-59.

33. Bopst L, Reinberg O, Lutz N. Femur fracture in preschool children: experience with flexible intramedullary nailing in 72 children. J Pediatr Orthop. 2007;27(3):299-303.

34. Chitgopkar SD. Flexible nailing of fractures in children using stainless steel Kirschner wires. J Pediatr Orthop B. 2008;17(5):251-5.

35. Flynn JM, Hresko T, Reynolds RA, Blasier RD, Davidson R, Kasser J. Titanium elastic nails for pediatric femur fractures: a multicenter study of early results with analysis of complications. J Pediatr Orthop. 2001;21(1):4-8.

36. Flynn JM, Luedtke L, Ganley TJ, Pill SG. Titanium elastic nails for pediatric femur fractures: lessons from the learning curve. Am J Orthop. 2002;31(2):71-4.

37. Luhmann SJ, Schootman M, Schoenecker PL, Dobbs MB, Gordon JE. Complications of titanium elastic nails for pediatric femoral shaft fractures. J Pediatr Orthop. 2003;23(4):443-7.

38. Narayanan UG, Hyman JE, Wainwright AM, Rang M, Alman BA. Complications of elastic stable intramedullary nail fixation of pediatric femoral fractures, and how to avoid them. J Pediatr Orthop. 2004;24(4):363-9.

39. Mann DC, Weddington J, Davenport K. Closed Ender nailing of femoral shaft fractures in adolescents. J Pediatr Orthop. 1986;6(6):651-5.

40. Volpon JB, Porto MR, Moretto M. Tratamento conservador das fraturas diafisárias do fêmur da criança. Rev Bras Ortop. 1997;32(1):11-4.

41. Simanovsky N, Tair MA, Removal of flexible titanium nails in children. J Bone Joint Surg Br. 2004;86:947-53

42. Schmalzried TP, Grogan TJ, Neumeier PA, Dorey FJ. Metal removal in a pediatric population: benign procedure or necessary evil? J Pediatr Orthop. 1991;11(1):72-6.

43. Alzahrani AG, Behairy YM, Alhossan MH, Arab FS, Alammari AA. Removal of internal fixation in pediatric patients. Saudi Med J. 2003;24(3):254-5.

44. Highland TR, LaMont RL. Deep, late infections associated with internal fixation in children. J Pediatr Orthop. 1985;5(1):59-64.

45. Lovell ME, Galasko CS, Wright NB. Removal of orthopedic implants in children: morbidity and postoperative radiologic changes. J Pediatr Orthop B. 1999;8(2):144-6.

46. Richards RH, Palmer JD, Clarke NMP. Observation on removal of metal implants. Injury. 1992;23(1):25-8.

47. Kahle WK. The case against routine metal removal. J Pediatr Orthop.1994; 14(2):229-37.

48. Peterson HA. Metallic implant removal in children. J Pediatr Orthop.2005; 25(1):107-15

49. Jago RD, Hindley CJ. The removal of metalwork in children. Injury.1998; 29(6):439-41 